\title{
Direct lateral retroperitoneal approach for the surgical treatment of lumbar discitis and osteomyelitis
}

\author{
Karthik Madhavan, M.D., ${ }^{1}$ Steven Vanni, D.C., D.O., ${ }^{1}$ and Seth K. Williams, M.D. ${ }^{2}$ \\ ${ }^{1}$ Department of Neurological Surgery, University of Miami Miller School of Medicine, Miami, Florida; and \\ ${ }^{2}$ Department of Orthopedics and Rehabilitation, University of Wisconsin School of Medicine and Public \\ Health, Madison, Wisconsin
}

Object. The medical management of discitis and osteomyelitis with long-term antibiotic therapy and bracing usually results in eradicated infection. Surgical management is appropriate when medical management fails and in some cases with pyogenic deformity or neurological deficit. The success of surgery depends on adequate debridement of the necrotic infected disc and vertebral body, along with anterior column reconstruction and vertebral stabilization. Debridement is typically performed via an anterior retroperitoneal approach, which can necessitate mobilization of the great vessels for proper exposure. Mobilization can be technically difficult and lead to vascular injury. The purpose of this study was to evaluate an alternative technique for the surgical treatment of lumbar discitis and osteomyelitis using a direct lateral retroperitoneal approach, which allows for thorough debridement and anterior column reconstruction while avoiding the need to mobilize the great vessels.

Methods. A retrospective chart analysis was performed for all patients who had presented with lumbar discitis and osteomyelitis and had undergone surgical management via the direct lateral retroperitoneal approach in the period from 2006 to 2013. Collected data included surgical blood loss, perioperative complications (wound infection, vascular injury, approach-related complications, and neurological injury), need for secondary procedures, microbiological and laboratory results, and efficacy of infection eradication. Imaging studies were reviewed as well.

Results. Ten patients, 7 male and 3 female, underwent this procedure at the authors' institution in the defined period. Average blood loss was $272 \mathrm{ml}$ (range $150-800 \mathrm{ml}$, with $800 \mathrm{ml}$ in the only 2-level case). There were no vascular injuries. Average follow-up was 680 days, although 4 patients did not complete the follow-up beyond 6 months. Eight patients underwent immediate posterior pedicle screw instrumentation. Two patients did not undergo posterior instrumentation, and one of these developed a kyphotic deformity that required a secondary posterior procedure. Infection was eradicated in all patients according to a history, physical examination, imaging studies, and laboratory parameters (complete blood count, erythrocyte sedimentation rate, and C-reactive protein). One patient developed a painful neuroma at the iliac crest harvest site, and one patient had a retroperitoneal hematoma. Otherwise, there were no approach-related neurological injuries or complications. Neither was there any postoperative surgical site infection.

Conclusions. The direct lateral approach for the surgical treatment of lumbar discitis and osteomyelitis allows for thorough debridement and spinal reconstruction without the need to mobilize the great vessels. This technique effectively eradicated infection in all cases, with reasonable blood loss and no vascular injuries. This approach should be considered as an alternative to the open anterior approach. The authors recommend posterior instrumentation to prevent the development of kyphosis.

(http://thejns.org/doi/abs/10.3171/2014.6.FOCUS14150)

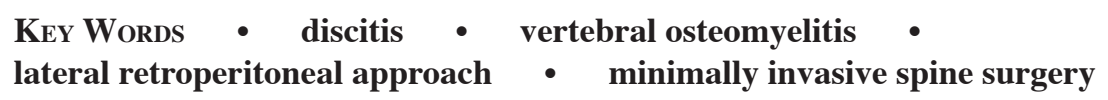

$\mathrm{D}$ ISCITIS and osteomyelitis accounts for approximately 1 case in 10,000 hospital admissions, and 30\% of cases are lumbar. ${ }^{15}$ Infection usually occurs as a result of hematological spread from another source but can also be iatrogenic due to an invasive spine procedure or caused by a penetrating injury. Medical management, which typically consists of intravenous antibiotic therapy for 6 weeks followed by 6 weeks or more of oral antibiotics, along with bracing, eradicates the infection in most cases. ${ }^{1,7}$ Surgical management is an option when medical

Abbreviations used in this paper: $\mathrm{CBC}=$ complete blood count; $\mathrm{CRP}=\mathrm{C}$-reactive protein; $\mathrm{ESR}=$ erythrocyte sedimentation rate. treatment fails or there is a neurological deficit, mechanical spinal instability along with deformity, or compromised immune system. ${ }^{22}$ The success of surgery depends on adequate debridement of the necrotic infected disc and vertebral body, followed by anterior column reconstruction and vertebral stabilization. While it is possible to approach the infection posteriorly, such an approach has limitations with respect to adequacy of debridement and reconstruction. Therefore, debridement is typically performed through an anterior retroperitoneal approach, which can necessitate mobilization of the great vessels for proper exposure. Mobilization can be technically difficult and result in vascular injury and, in some cases, massive blood loss. We pres- 
ent an alternative technique for the surgical treatment of lumbar discitis and osteomyelitis using a direct lateral retroperitoneal approach, which allows for thorough debridement and anterior column reconstruction while avoiding the need to mobilize the great vessels.

\section{Methods}

We performed a retrospective chart analysis of all the patients who had presented with lumbar discitis and osteomyelitis at L1-2, L2-3, L3-4, and/or L4-5 and had undergone surgical management via the direct lateral retroperitoneal approach in the period from 2006 to 2013 at our institution. Collected data included surgical blood loss, perioperative complications (wound infection, vascular injury, approach-related complications, and neurological injury), need for secondary procedures, microbiological and laboratory results, and efficacy of infection eradication. Imaging studies were reviewed as well. We obtained institutional review board approval to perform this study.

\section{Results}

Ten patients, 7 male and 3 female, underwent this procedure (S.K.W. or S.V.). The average patient age was 70 years. Medical management with at least one course of intravenous antibiotics for 6 weeks had failed in every patient, with each having persistent infection according to imaging studies, laboratory values, and progressive back pain. Preoperatively, 6 of the 10 patients had weakness of the hip flexor muscles, graded 3 or 4 out of 5 and thought to be caused by iliopsoas abscess and/or iliopsoas atrophy due to proximity to the infected area. There was minimal or no epidural component to the infection and no focal neurological deficit due to epidural abscess. Debridement of the entire disc space and adjacent vertebral bodies was possible through the direct lateral retroperitoneal approach. Structural iliac crest autograft bone was used for anterior column reconstruction except in one patient with a large bony defect, in whom a titanium mesh cage with morselized autograft iliac crest was used. The infection involved one disc and the adjacent vertebral body in 7 cases (L2-3 in 3 cases, L3-4 in 3 cases, and L1-2 in 1 case). One case involved 2 levels (L3-4 and L4-5), and 2 cases involved L2-3 initially and then later L4-5. Average blood loss was $272 \mathrm{ml}$ (range 150-800 ml, with $800 \mathrm{ml}$ in the only 2-level case). There were no vascular injuries. Microbiological analysis revealed Staphylococcus aureus in two patients and Enterococcus faecalis and Escherichia coli in one patient each. Six patients did not grow any organism. Average follow-up was 680 days, although 4 patients did not complete the follow-up beyond 6 months. Eight patients underwent immediate posterior pedicle screw instrumentation, all placed percutaneously except in one patient who required posterior osteotomies for kyphosis correction. Posterior instrumentation was limited to the involved segments in 5 cases and extended beyond the involved segments in 3 cases because the surgeon deemed this necessary to reestablish spinal stability. Instrumentation was performed on the same day

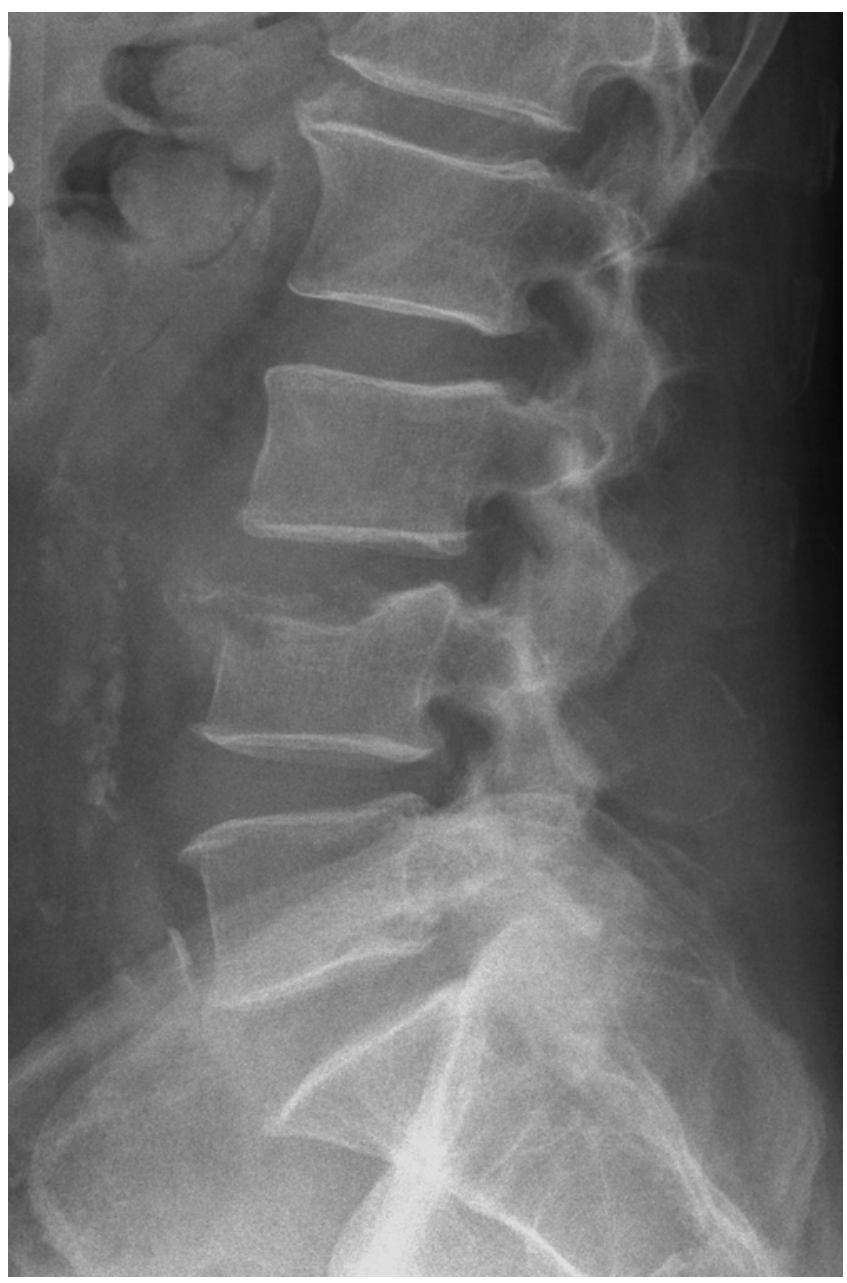

FIG. 1. Preoperative lumbar spine lateral radiograph demonstrating L3-4 discitis and osteomyelitis, with erosion of the superior endplate of L-4.

as the discitis and osteomyelitis debridement and reconstruction. Two patients did not undergo posterior instrumentation, and one of these developed a kyphotic deformity that required a secondary posterior procedure. Two patients developed discitis and osteomyelitis two levels below the original L2-3 level, requiring further surgery at L4-5. Infection was eradicated in all patients according to a history, physical examination, imaging studies, and laboratory parameters (complete blood count [CBC], erythrocyte sedimentation rate [ESR], and C-reactive protein [CRP]). Two patients were kept on long-term suppressive oral antibiotics under the direction of infectious disease specialists. One patient developed a painful neuroma at the iliac crest harvest site, and one patient with a myeloproliferative disorder and underlying coagulopathy postoperatively developed a retroperitoneal hematoma that was self-limited and did not require intervention. All 6 patients with hip flexor weakness before surgery improved postoperatively, and we could not identify any patients with persistent or worsening hip flexor weakness because of the approach. One patient had decreased sensation to light touch on the anterior thigh on the approach side, which resolved by 6 months. Otherwise, however, 


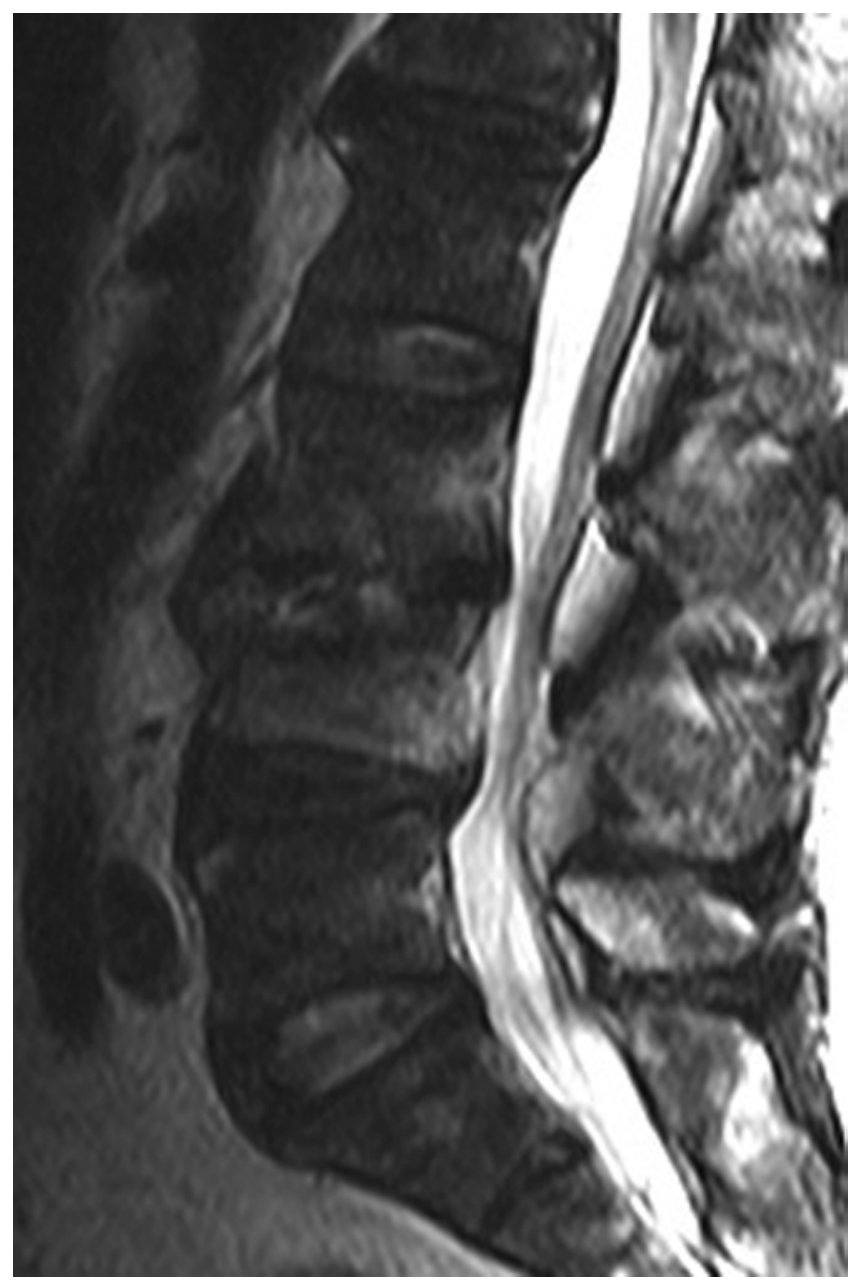

FIG. 2. Preoperative lumbar spine sagittal T2-weighted MR image demonstrating L3-4 discitis and osteomyelitis.

there were no approach-related neurological injuries or complications. Neither was there any postoperative surgical site infection, either from the lateral approach or the posterior instrumentation.

\section{Discussion}

In 1911, the pre-antibiotic era, Russell Hibbs performed the first spine fusion in a tuberculous spine after debridement of infected tissue and placement of autologous bone. ${ }^{14}$ The goals of discitis and osteomyelitis surgery are eradication of the infection and the creation of an environment in which robust fusion can occur at that level. ${ }^{22}$ To achieve these goals, it is important to debride all necrotic disc and bone, leaving only viable vertebral body. A structural interbody graft can then be placed to achieve fusion. Vertebral infections in the cervical and thoracic spine can be surgically treated without the need to manipulate major blood vessels. Proper exposure of L1-2 through L4-5 typically requires manipulation of the peritoneal contents, which can lead to ileus. More importantly, however, it often requires dissection of the vena cava, aorta, or iliac vessels from their position directly adjacent to the lumbar spine. Because of scarring and

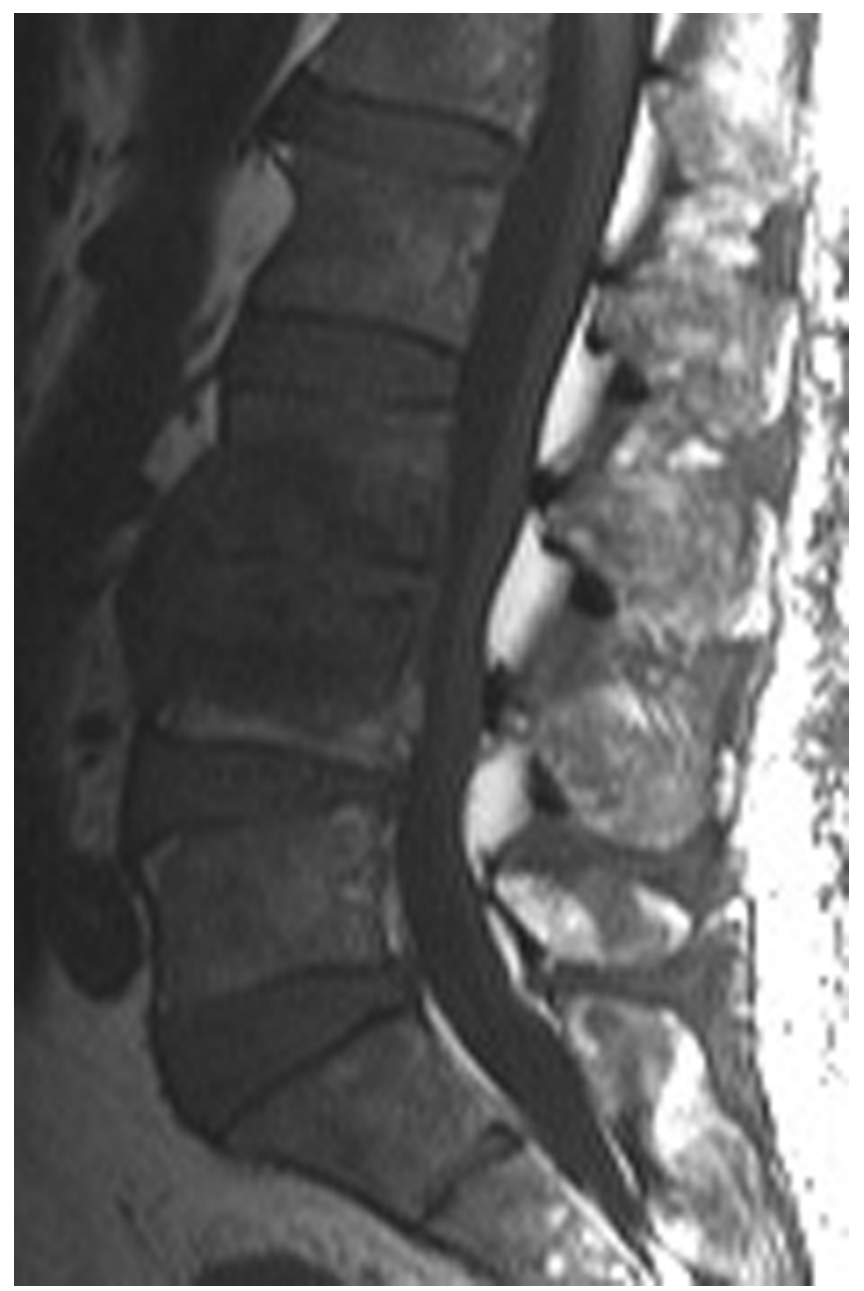

FIG. 3. Preoperative lumbar spine sagittal T1-weighted MR image demonstrating L3-4 discitis and osteomyelitis.

the anatomical distortion due to infection, dissection can result in blood vessel injury and massive blood loss..$^{10,11}$ The approach to L1-2, L2-3, and L3-4 requires a greater degree of blood vessel mobilization than the approach to L4-5 and L5-S1, although the iliac vessels can also cross the L4-5 disc space anteriorly. ${ }^{2,12,13,16,21}$

The direct lateral lumbar retroperitoneal approach has the advantage of excellent exposure for thorough debridement and reconstruction, without the need for blood vessel mobilization or manipulation of the peritoneal contents. ${ }^{5,17,20}$ The surgical approach we used is identical to the lateral retroperitoneal approach for interbody fusion that is gaining in popularity for the treatment of lumbar stenosis with degenerative scoliosis and/or spondylolisthesis. Figures 1-8 were obtained from the same patient, a 64-yearold overweight male smoker with poorly controlled diabetes whose condition worsened despite 6 weeks of intravenous antibiotic therapy. Figures 1-3 show a preoperative lateral radiograph and sagittal T1- and T2-weighted MRI sequences demonstrating L3-4 discitis and osteomyelitis. The intraoperative fluoroscopy image in Fig. 4 shows debridement of the infection performed through a tablemounted retractor via a lateral approach. Once debridement was complete, we used sequential trial implants from 


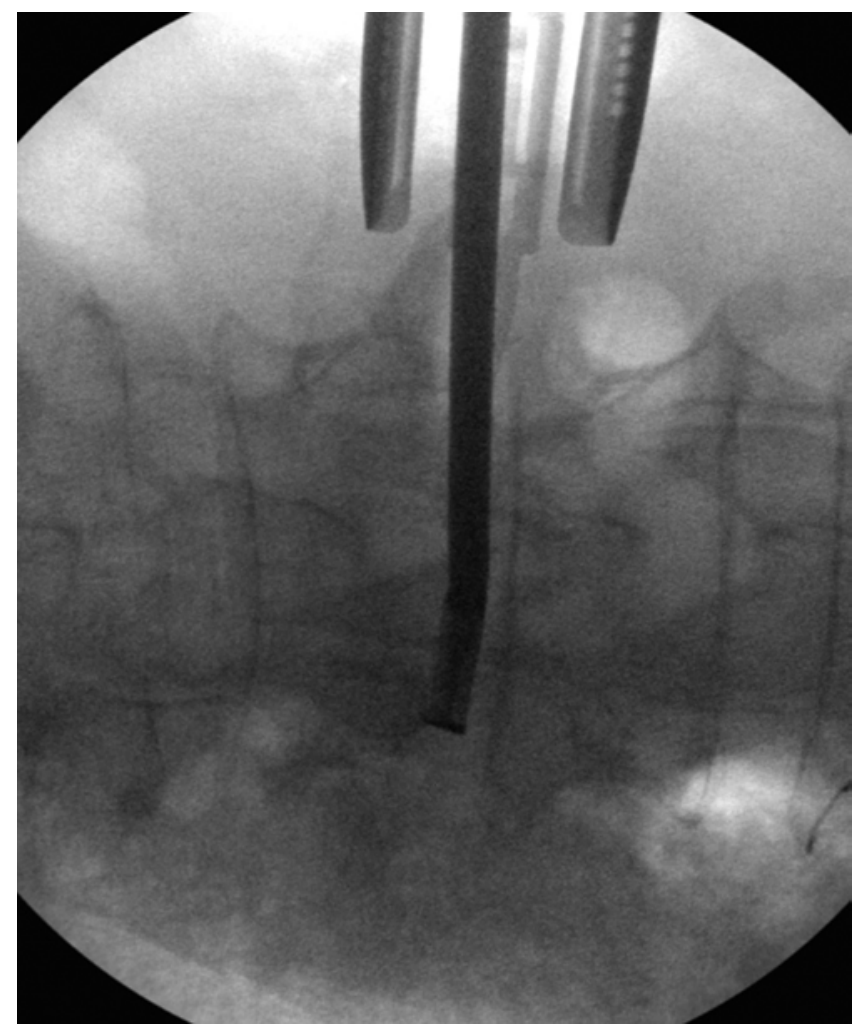

FIG. 4. Intraoperative anteroposterior fluoroscopic image, with the patient in the lateral position, showing debridement of the L3-4 disc space and adjacent vertebral bodies with a curette through the tablemounted retractor.

a standard lateral approach fusion tray (equipment from either NuVasive Inc. or DePuy Synthes Spine) to determine the size of autograft bone required for structural support. Tricortical iliac crest was then harvested, either through the same incision in the lower lumbar spine or through a separate incision, cut to match the size of the trial implant, and then placed in a manner to achieve as much of an interference fit as possible. Figure 5 shows placement of the structural iliac crest autograft. Debridement resulted in a void that was usually not uniform, so the degree of interference fit was variable. We attempted to fill as much of the void as possible with autograft, although we did not place morselized autograft in addition to structural graft. We did not have any iliac crest wound infections despite harvesting the graft after debridement. Figure 6 shows an immediate postoperative coronal CT scan, and Figs. 7 and 8 are radiographs taken 2 years after surgery, showing a solid mature arthrodesis at L3-4 with the infection eradicated. Note that healing occurred not just across the bone graft but peripherally as well, and this peripheral bone formation was typically the first radiographic sign of eradicated infection and successful arthrodesis. We believe that this peripheral bone healing is the reason arthrodesis occurs despite variable degrees of structural autograft interference fit. Figure 9 is a coronal CT scan obtained in a different patient 3 years after surgery, demonstrating the healing that occurs around the bone graft in the disc space periphery. We chose structural iliac crest autograft to add as much stability as possible, but because of this new bone forma-

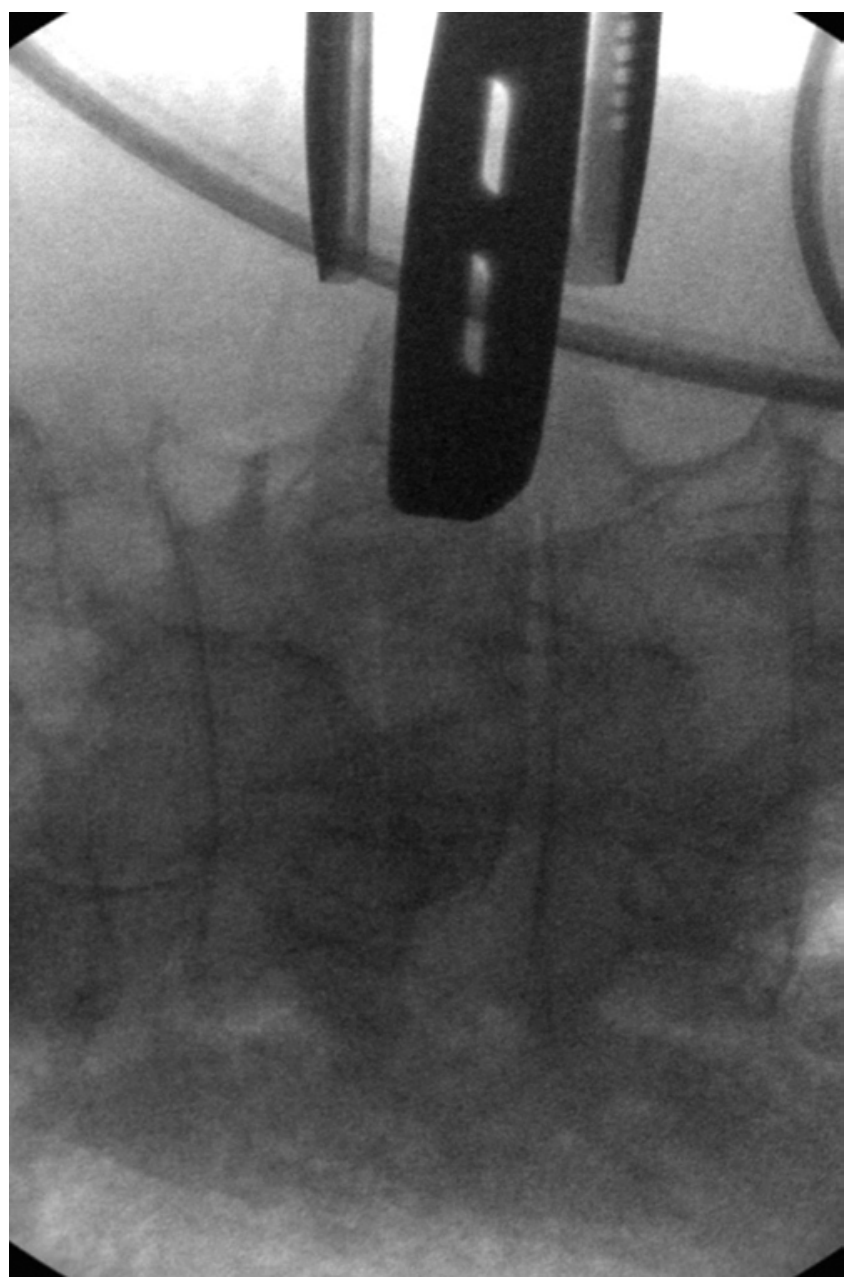

FIG. 5. Intraoperative anteroposterior fluoroscopic image, again with the patient in the lateral position, showing placement of the structural iliac crest autograft through a table-mounted retractor. An interbody spacer trial was used to advance the graft into the L3-4 interbody space.

tion in the disc space periphery, it is possible that healing would have occurred if morselized graft had been used instead of structural graft.

Consideration can be given to reconstructing the spine anteriorly with structural bone graft alone without instrumentation, as is described in degenerative cases, although this is typically done with a structural cage in the setting of a competent annulus and endplates. ${ }^{9,19} \mathrm{We}$ believe that the infected spine is more inherently unstable than the typical degenerative spine, so instrumentation should be strongly considered. One of the two patients who did not undergo posterior instrumentation developed a kyphotic deformity that later required posterior surgery. Lateral fixation can theoretically be performed using plates and screws through the same incision, but there may not be enough residual healthy vertebral body to accommodate the screws. We believe this construct is biomechanically inferior to posterior pedicle instrumentation and also places instrumentation in the infected area. ${ }^{18}$ Patients were repositioned and a fresh sterile setup was used for posterior instrumentation. We performed 


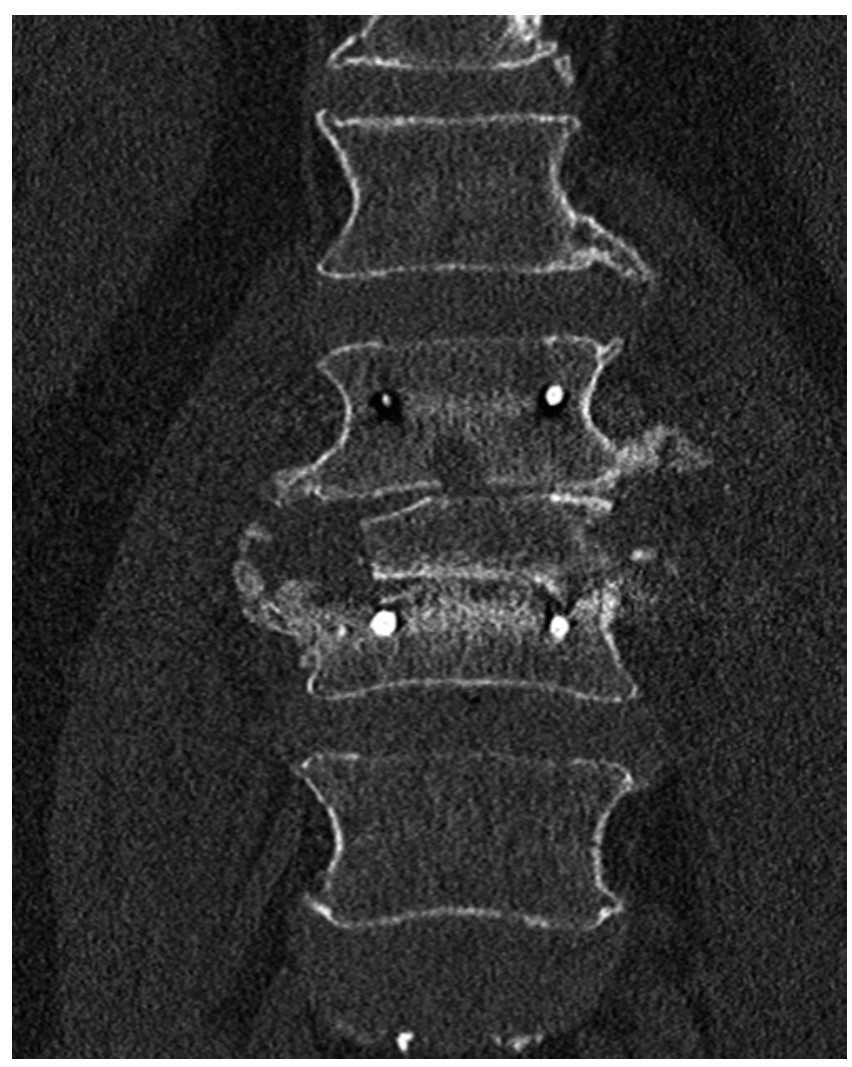

FIG. 6. Immediate postoperative lumbar spine coronal CT scan demonstrating placement of the structural iliac crest autograft at L3-4.

the lateral debridement and posterior instrumentation at the same setting, although the procedures can be staged. Ideally, instrumentation is limited to the involved levels, aiming the screws into healthy vertebral body, although it may need to be extended to other levels in cases in which screws cannot be safely placed into the involved vertebral body or if the patient has poor bone quality. Removing the instrumentation or shortening the construct can be considered once the fusion is mature, but we did not find either necessary in our series.

Surgeons who are comfortable with the direct lateral retroperitoneal approach for degenerative pathology should exercise caution when adapting this approach to infectious cases. Local anatomy is often distorted by the infection, and the disc space may not be readily identifiable because it is necrotic and inadequate. The distorted anatomy makes it easier to stray from the disc space and encounter bleeding from a segmental vessel or the great vessels. If there is iatrogenic injury to a segmental vessel, aorta, vena cava, or iliac vessels, it can be difficult to control the bleeding through the small incision used for the lateral approach. Advance preparations should be made to extend the incision widely and call for assistance from a vascular surgeon in the event that such an injury occurs. The dilators may plunge into the soft necrotic spinal column and lead to neurological injury, so great care must be taken to carefully position the dilators and retractor. Fluoroscopy should be carefully set up to obtain reliable anteroposterior and lateral views and used frequently during the procedure to maximize safety. Preoperative MRI and

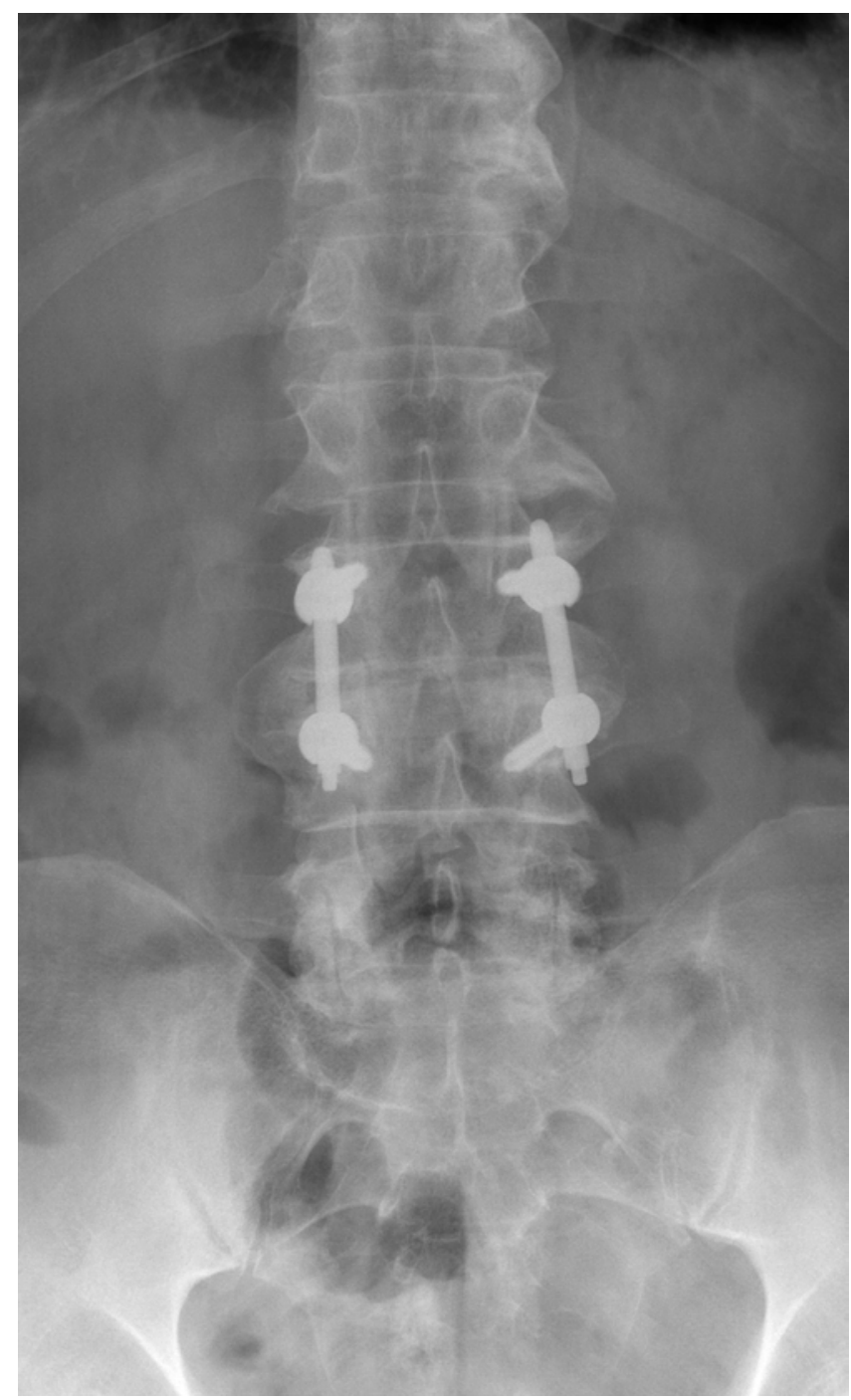

FIG. 7. Lumbar spine anteroposterior radiograph taken 2 years after surgery, showing a solid mature arthrodesis at L3-4 with the infection eradicated. Note the bridging bone around the periphery of the L3-4 disc space. Percutaneous pedicle screws were used at L3-4.

CT studies should be closely scrutinized to plan the surgical corridor. Other surgical risks are similar to those for the lateral approach for degenerative pathology, including iatrogenic nerve injury causing weakness and/or sensory deficits in the leg on the approach side, hip pain, and bowel and visceral injury. 3,4,6,8 The infection-altered anatomy did not appear to increase the approach-related morbidity any more than with the typical elective degenerative spine lateral approach, but it is difficult to compare the patients undergoing these procedures because those with infection are often quite debilitated and have preexisting hip flexor muscle weakness due to deconditioning and iliopsoas proximity to the site of infection.

Postoperative care does not differ substantially from that following a typical open anterior approach protocol. Given the minimally invasive nature of the procedure, emphasis is placed on early mobilization, with or without a brace depending on personal preference. A regular diet is typically well tolerated immediately after surgery. Intra- 


\section{K. Madhavan, S. Vanni, and S. K. Williams}

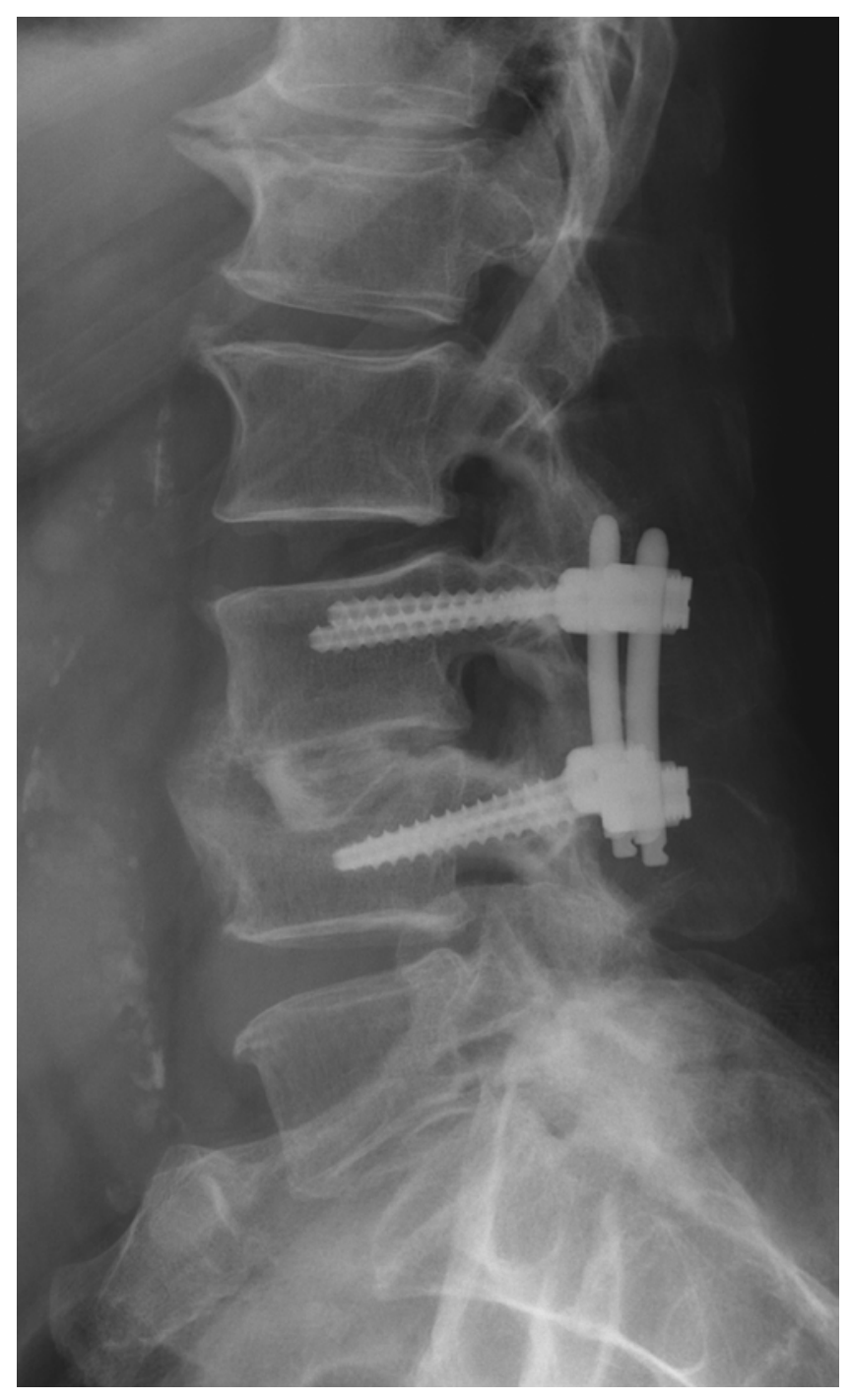

FIG. 8. Lumbar spine lateral radiograph taken 2 years after surgery, showing a solid mature arthrodesis at L3-4 with the infection eradicated. Note the bridging bone anterior to the structural bone graft in addition to graft incorporation. Percutaneous pedicle screws were used at L3-4.

venous antibiotics are administered for 6 weeks, followed by oral antibiotics for at least 6 weeks, until the CRP is normal or near normal, the ESR is trending down, there is substantial clinical improvement, and imaging studies show evidence of fusion across the previously infected area. In certain patients with a high risk of recurrence, one should consider long-term oral antibiotic suppression.

The main deficiencies of this study are the retrospective data collection and the lack of a control group. Future prospective data collection is appropriate. Given our experience, we believe the lateral approach is safer and at least as effective as the anterior approach. Thus, we do not think a randomized controlled trial is appropriate. We did not perform any open anterior surgeries for discitis and osteomyelitis at L1-2, L2-3, L3-4, or L4-5 during the study period. The role of the lateral approach in the setting of a significant epidural abscess is unclear. Another limitation is a potential conflict of interest for

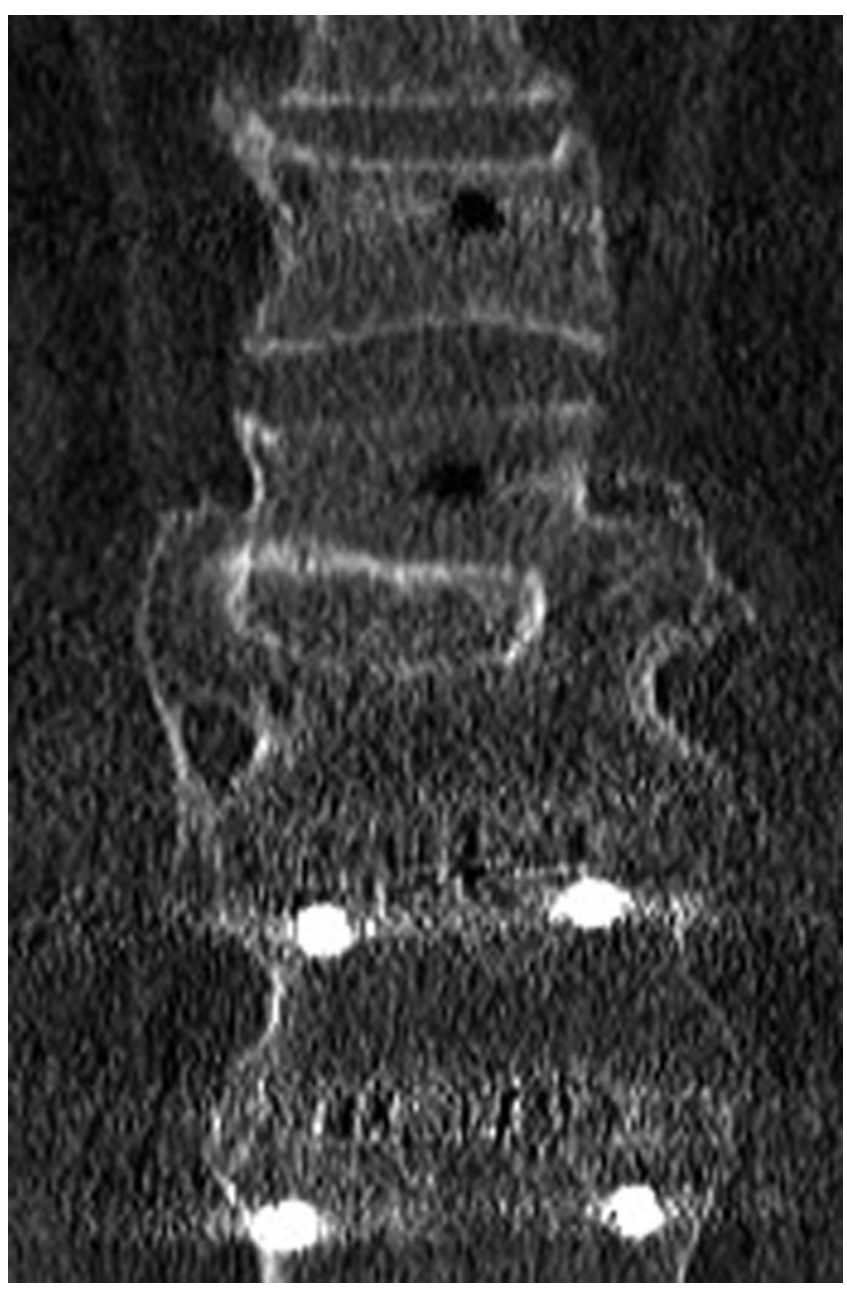

FIG. 9. Coronal lumbar spine CT scan obtained in a 60-year-old man 3 years after surgery, showing new bone formation peripherally around the structural graft in the previously infected disc space.

the senior author, who serves as a consultant for DePuy Synthes Spine, which manufactures the equipment used in some of the reported cases.

\section{Conclusions}

The minimally invasive direct lateral retroperitoneal approach for surgical treatment of lumbar discitis and osteomyelitis allows for thorough debridement and spinal reconstruction without the need to mobilize the great vessels. This technique allowed effective eradication of infection in all cases, with reasonable blood loss, minimal approach-related morbidity, and no vascular injuries. The approach should be considered as an alternative to the open anterior approach. We recommend posterior instrumentation, either percutaneous or open depending on the circumstances, to prevent the development of kyphosis.

\section{Disclosure}

Dr. Williams is a consultant for DePuy Synthes Spine.

Author contributions to the study and manuscript preparation include the following. Conception and design: Williams, Vanni. Acquisition of data: all authors. Analysis and interpretation of data: 


\section{Lateral retroperitoneal approach for discitis and osteomyelitis}

all authors. Drafting the article: Williams, Madhavan. Critically revising the article: Williams. Reviewed submitted version of manuscript: Williams, Madhavan. Approved the final version of the manuscript on behalf of all authors: Williams. Statistical analysis: Williams, Madhavan. Study supervision: Williams.

\section{References}

1. Afshar M, Reilly RF: Spondylodiscitis in a patient on chronic hemodialysis. Nat Rev Nephrol 7:599-604, 2011

2. Baker JK, Reardon PR, Reardon MJ, Heggeness MH: Vascular injury in anterior lumbar surgery. Spine (Phila Pa 1976) 18:2227-2230, 1993

3. Banagan K, Gelb D, Poelstra K, Ludwig S: Anatomic mapping of lumbar nerve roots during a direct lateral transpsoas approach to the spine: a cadaveric study. Spine (Phila Pa 1976) 36:E687-E691, 2011

4. Benglis DM, Vanni S, Levi AD: An anatomical study of the lumbosacral plexus as related to the minimally invasive transpsoas approach to the lumbar spine. Laboratory investigation. J Neurosurg Spine 10:139-144, 2009

5. Berjano P, Balsano M, Buric J, Petruzzi M, Lamartina C: Direct lateral access lumbar and thoracolumbar fusion: preliminary results. Eur Spine J 21 (Suppl 1):S37-S42, 2012

6. Brier-Jones JE, Palmer DK, Ǐnceoğlu S, Cheng WK: Vertebral body fractures after transpsoas interbody fusion procedures. Spine J 11:1068-1072, 2011

7. Butler JS, Shelly MJ, Timlin M, Powderly WG, O’Byrne JM: Nontuberculous pyogenic spinal infection in adults: a 12-year experience from a tertiary referral center. Spine (Phila Pa 1976) 31:2695-2700, 2006

8. Cahill KS, Martinez JL, Wang MY, Vanni S, Levi AD: Motor nerve injuries following the minimally invasive lateral transpsoas approach. Clinical article. J Neurosurg Spine 17:227231,2012

9. Cappuccino A, Cornwall GB, Turner AW, Fogel GR, Duong HT, Kim KD, et al: Biomechanical analysis and review of lateral lumbar fusion constructs. Spine (Phila Pa 1976) 35 (26 Suppl):S361-S367, 2010

10. Christensen FB, Bünger CE: Retrograde ejaculation after retroperitoneal lower lumbar interbody fusion. Int Orthop 21: 176-180, 1997

11. Fantini GA, Pappou IP, Girardi FP, Sandhu HS, Cammisa FP Jr: Major vascular injury during anterior lumbar spinal surgery: incidence, risk factors, and management. Spine (Phila Pa 1976) 32:2751-2758, 2007

12. Gumbs AA, Bloom ND, Bitan FD, Hanan SH: Open anterior approaches for lumbar spine procedures. Am J Surg 194:98102,2007
13. Gumbs AA, Hanan S, Yue JJ, Shah RV, Sumpio B: Revision open anterior approaches for spine procedures. Spine J 7:280285,2007

14. Heary RF, Madhavan K: The history of spinal deformity. Neurosurgery 63 (3 Suppl):5-15, 2008

15. Jones NS, Anderson DJ, Stiles PJ: Osteomyelitis in a general hospital. A five-year study showing an increase in subacute osteomyelitis. J Bone Joint Surg Br 69:779-783, 1987

16. Kozak JA, Heilman AE, O’Brien JP: Anterior lumbar fusion options. Technique and graft materials. Clin Orthop Relat Res (300):45-51, 1994

17. Laws CJ, Coughlin DG, Lotz JC, Serhan HA, Hu SS: Direct lateral approach to lumbar fusion is a biomechanically equivalent alternative to the anterior approach: an in vitro study. Spine (Phila Pa 1976) 37:819-825, 2012

18. Le TV, Smith DA, Greenberg MS, Dakwar E, Baaj AA, Uribe JS: Complications of lateral plating in the minimally invasive lateral transpsoas approach. Clinical article. J Neurosurg Spine 16:302-307, 2012

19. Marchi L, Abdala N, Oliveira L, Amaral R, Coutinho E, Pimenta L: Stand-alone lateral interbody fusion for the treatment of low-grade degenerative spondylolisthesis. ScientificWorldJournal 2012:456346, 2012

20. Ozgur BM, Aryan HE, Pimenta L, Taylor WR: Extreme Lateral Interbody Fusion (XLIF): a novel surgical technique for anterior lumbar interbody fusion. Spine J 6:435-443, 2006

21. Raney FL, Adams JE, King D: Anterior lumbar disc excision and interbody fusion used as salvage procedure. J Bone Joint Surg Am 45:667-668, 1963

22. Vollmer DG, Tandon N: Infections of the spine, in Winn HR (ed): Youmans Neurological Surgery, ed 6. Philadelphia: Elsevier Saunders, 2012, Vol 4, pp 2831-2847

Manuscript submitted April 15, 2014.

Accepted June 16, 2014.

Portions of this work were presented in poster form at the 2013 Annual Meeting of the AANS/CNS Section on Disorders of the Spine and Peripheral Nerves held in Phoenix, AZ, on March 6-9, 2013.

Please include this information when citing this paper: DOI: 10.3171/2014.6.FOCUS14150.

Address correspondence to: Seth K. Williams, M.D., University of Wisconsin School of Medicine and Public Health, Department of Orthopedics and Rehabilitation, UWMF Centennial Building, 1685 Highland Ave., Madison, WI 53705. email: swilliams@ortho.wisc. edu. 